\title{
Isolated Scapula Body Fracture with Intrathoracic Displacement: Description of a New Pattern of Injury
}

\author{
Ismael Auñón-Martín, MD, PhD*, Carlos Olaya-González and Gema Muñoz Sánchez
}

Trauma Unit, Trauma and Orthopedic Surgery Service, Hospital 12 Octubre, Madrid, Spain

\begin{abstract}
Scapula fractures are relatively rare. Those that affect the body of the scapula account for approximately $50 \%$ of all scapula fractures. The indication of surgical treatment in the fractures of the body of the scapula is controversial. One of the indications for surgery is the intrathoracic displacement of a bone fragment. Within the low incidence of this complication, homogeneous anatomical patterns always related to high energy trauma have been described.
\end{abstract}

We present a case of fracture of the body of the scapula that required surgical treatment due to intrathoracic displacement of a bone fragment related to a low energy trauma, representing an anatomical pattern not described in the literature previously.

\section{Keywords}

Scapula fracture, Thoracic displacement, Complication

\section{Introduction}

The fractures of the scapula have an annual incidence of approximately $10 / 10^{5}$, therefore although the fractures of the scapula body represent between $35-52 \%$ of the total, those remain uncommon injuries [1-3]. Fractures of the scapular body usually occur in relation to high-energy trauma, and they are commonly associated with local and systemic injuries $[2,3]$. This fact has anatomical explanation, as the scapula is anatomically protected by the rib cage and the surrounding musculature, which also explains that, usually, the fractures of the body of the scapula tend to be slightly displaced.

The surgical indication in scapula body fractures using criteria of anatomical deformity is controversial [4]. Only in the presence of complications such as open fracture or neurovascular injury, the indications are clearer.

An unusual complication that implies a surgical indication in these fractures is the intrathoracic displacement of bone fragments. This condition is rare, especially in adults, and has been described occasionally in the bibliography, presenting well-defined fracture patterns [5-8]. In this case report, we describe a clinical case of scapular body fracture, as an isolated injury, with intrathoracic penetration of a fracture fragment with a morphology not previously described and which, in addition, occurs after a low energy trauma.

\section{Case Presentation}

A 32-year-old male is referred to our center, from an emergency service at a sky center, after an accident practicing snow board. He described a low energy trauma, falling from his height in abduction of his left shoulder while he was snowboarding downhill at low speed. He had pain in his left shoulder and functional impairment. In the physical examination the patient focused the pain on the distal clavicle, with local deformity at the acromioclavicular (AC) joint, and elevation of its distal end. He also presented diffuse pain on trapezius muscle. Neurovascular exploration was described as normal.

The patient had no medical-surgical history of interest, except a fracture of the contralateral clavicle that was operated 18 months ago and healed uneventfully.

In the emergency department (ED) a simple AP shoulder $x$-ray was performed, a grade III AC dislocation was diagnosed associated with a non-displaced longitudinal fracture of the body of the scapula (Figure $1 \mathrm{~A}$ and Figure 1B). Chest X-Ray was also obtained, and reported as normal. Presented in clinical session, orthopedic treatment was decided prescribing a sling.

*Corresponding author: Ismael Auñón-Martín, MD, PhD, Trauma Unit, Trauma and Orthopedic Surgery Service, Trauma, Hospital 12 Octubre, Madrid, Spain, Tel: (0034)-913-908-227

Accepted: May 16, 2019

Published online: May 18, 2019

Citation: Auñón-Martín I, Olaya-González C, Sánchez GM (2019) Isolated Scapula Body Fracture with Intrathoracic Displacement: Description of a New Pattern of Injury. J Orthop Rheumatism 3(1):47-50 


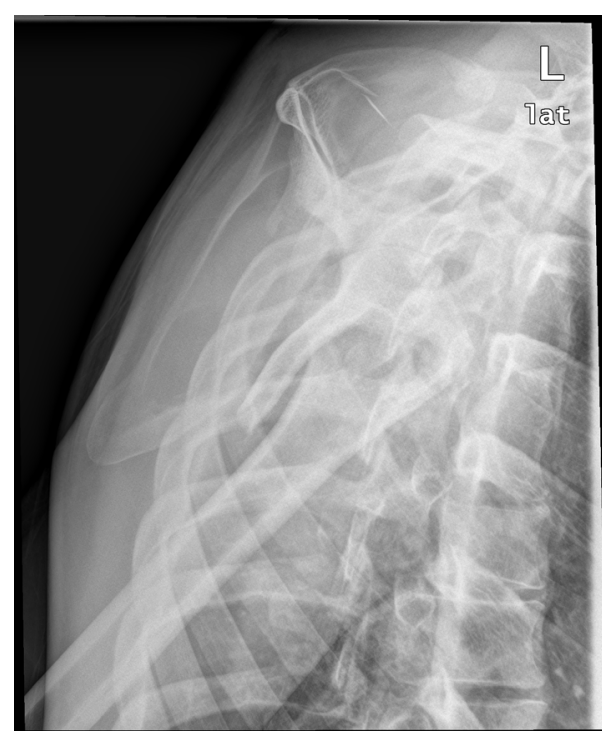

A

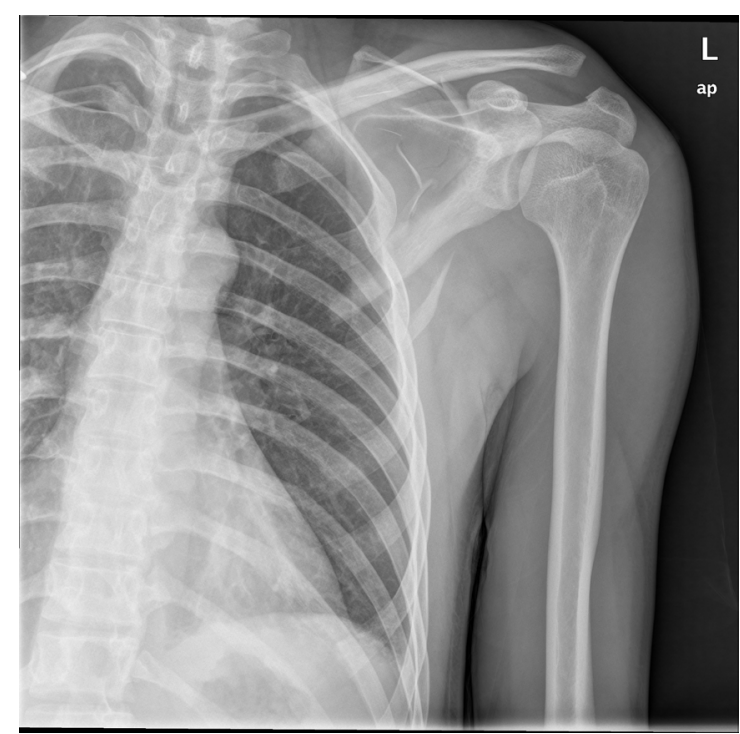

B

Figure 1: A) Transtoracic radiographic projection, Preoperative; B) Anteroposterior radiographic projection, Preoperative.

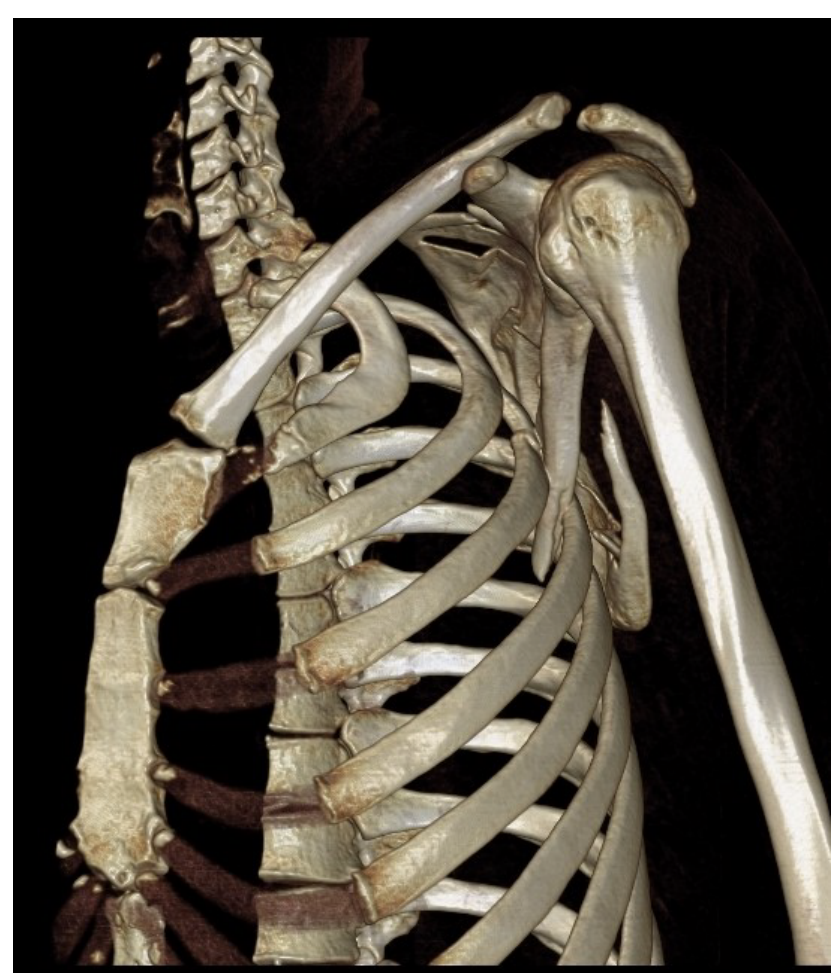

Figure 2: TAC 3D Reconstruction, Preoperative.

The patient returned to the ED 48 hours later due to increasing pain, on the clavicle and progressive blocking of the shoulder mobility. In the physical examination, the patient had an elevated shoulder, with locking of the shoulder rotations. The scapular mobility was absent over the thoracic wall. A CT Scan was performed (Figure 2).

CT scan showed a partial longitudinal fracture of the lateral edge of the scapula, with the lateral proximal fragment introduced between the third and fourth ribs, without lung injuries, or associated rib fractures. The medial border of the scapula presented continuity throughout its length. The AC joint presented only a mild displacement.

Surgical intervention was decided, in collaboration with thoracic surgeons. With the patient in lateral decubitus, a dorsolateral thoracotomy was performed by an incision parallel to the lower angle of the scapula. The latissimus dorsi muscle was partially sectioned, dissecting the anterior serratus to locate the intrathoracic fragment of the scapula, which was in the third intercostal space with no rib fracture associated. The collapse of the lung was performed by anesthesia team, extracting the fragment in maximum abduction of the shoulder and bone traction. The AC joint deformity was reduced after fragment extraction. Despite the fact a lung injury was not observed, a thoracic drainage was placed in the $5^{\text {th }}$ intercostal space, in the middle mamillar line, closing the third space by pericostal cerclage to diminish the possibility of recurrence.

The cranial fragment of the lateral border of the scapula that presented the intrathoracic displacement was of a limited thickness and showed a tendency to reintroduce itself into the defect of the thoracic wall with the scapulothoracic movements. So with these findings it was decided to resect this fragment. On the other hand, the caudal fragment of the lateral border did not show the capacity to enter the chest wall gap and was regularized. Since the scapula fracture was incomplete and showed good stability, osteosynthesis was not considered necessary, obtaining a complete range of motion of scapulothoracic joint. Wound closure was performed and the arm was placed on a sling in a neutral position.

The immediate postoperative period had no incidences. The AC joint presented only a moderate deformity and the patient tolerated the onset of mobility without pain. The thoracic drainage was removed after 48 hours. Passive and assisted mobilization of the shoulder was allowed 24 hours 


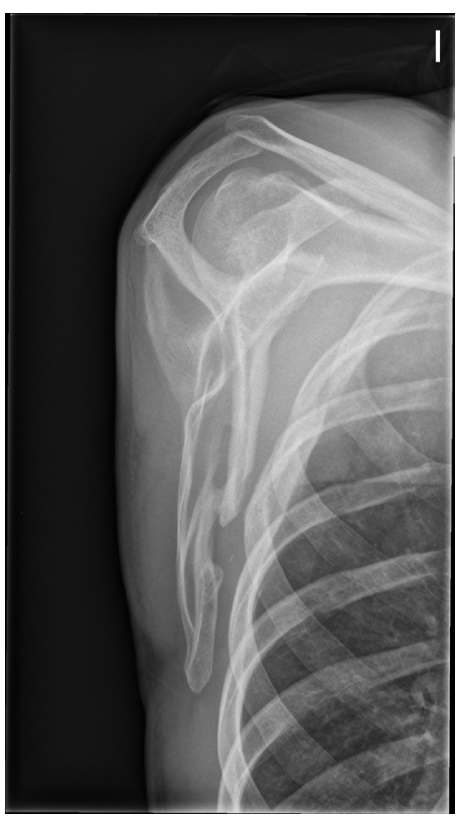

A

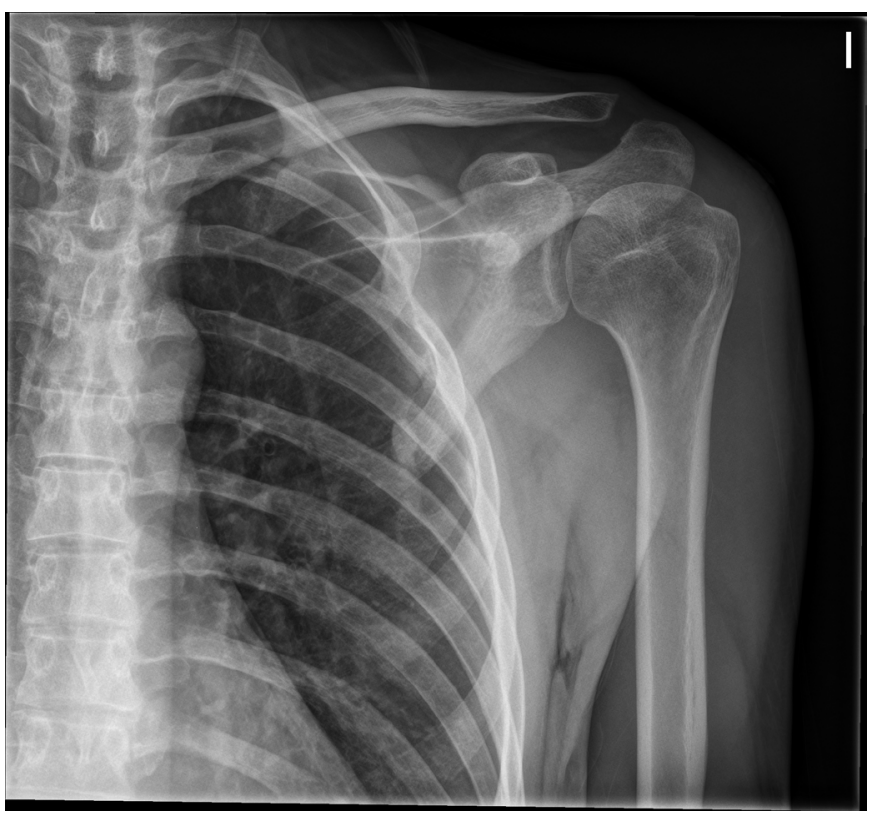

B

Figure 3: A) Radiographic projection in Y of scapula, Postoperative; B) Anteroposterior radiographic projection, Postoperative.

after surgery. Preoperative shoulder lock and pain were reversed, and the AC joint anatomy was restored.

After eight weeks, the fracture was radiologically healed. He resumed sports activity at three months. At 6 months the patient had no limitation for his work or sports activity, and in the physical examination the shoulder and scapulothoracic ranges of motion were comparable to the opposite upper limb (Figure 3A and Figure 3B).

\section{Discussion}

Scapula fractures are report as rare in the literature. They have been shown to occur with a rate of $1 \%$ of all fractures, $5 \%$ of the shoulder girdle.

These injuries are typically associated with high energy trauma. $96 \%$ of the scapula fractures have other injuries involved [1-3], being rib fractures and lung injuries the most commonly injuries associated $[2,3]$.

Body scapula fractures are about $35-50 \%$ of all scapula fractures, ant their management is still controversial $[2,3]$.

Surgical indication based on their morphology has been developed during the last years. Some surgical criteria like medialization $>20 \mathrm{~mm}$, angular deformity $\geq 45^{\circ}$ on a scapular Y shoulder radiograph and a glenopolar angle $<22^{\circ}$, have been proposed to improved functional results [4]. On the other hand, we still don't have enough scientific evidence regarding surgical indication. Zlowodzki, et al. published a large series (more than 500 cases), where $99 \%$ of these fractures where body scapula fractures treated non-operatively, finding good results in $86 \%$ of cases $[9,10]$. Thus, the surgical treatment of these fractures should be individualized, and not only be based on deformity parameters $[9,10]$.

There are other parameters in order to treat these frac- tures surgically, e.g. the presence of complications as open injuries, neurovascular compromise or scapulothoracic dissociation. One specific complication could be the intrathoracic displacement of a bone fragment or the scapula itself. This intrathoracic displacement has been described in 4 adult patients. In 2 patients, the intrathoracic fragment was the inferior scapula angle, and the other 2 cases had a transverse body fracture and the intrathoracic fragment was the lateral border of the distal fragment [5-8]. All 4 patients suffered traffic accidents, and they had thoracic injuries associated.

The case presented in this case report has some differences from other published cases.

It is remarkable that this patient had a low energy trauma, compared to the rest of the patients in other case reports, who suffered traffic accidents. The absence of associated injuries should be noticed as well, as the other cases had also thoracic injuries. The only associated injury in this case was a AC dislocation, probably associated with the initial displacement of the fracture, as the joint was spontaneously reduced after fragment extraction.

Finally, the intrathoracic fragment was the lateral fragment of the proximal segment, in contrast with the other cases published in adult population.

The delay in diagnosis of intrathoracic displacement is significant in the present case. This delay has been described previously [5], and it is associated to the low sensitivity of plain $x$-rays to evaluate the intrathoracic displacement of the scapula. It is also notable the importance of clinical findings, as scapula elevation or the scapulothoracic movement blocking. Despite there are several scapula x-ray studies described, the gold standard in diagnose scapula fractures and their complications is the CT scan. 
The recommended treatment of these cases is also varied. Despite the surgical treatment is indicated, there has been described cases of closed reduction, open reduction through Judet approach or even thoracotomy. We cannot stablish conclusions regarding the best treatment option, but in case of delayed diagnosis, and following a previous report, we decided to perform a thoracotomy in order to treat the possible complications extracting the intrathoracic fragment [5]. Only one case previously published required fracture osteosynthesis. In our case, after reducing the dislocation, the partial resection of the fragment restored the normal scapulothoracic movement, and no other surgical technique [6] was needed regarding the scapula fracture fixation.

\section{References}

1. Ideberg R, Grevsten S, Larsson S (1995) Epidemiology of scapular fractures. Incidence and classification of 338 fractures. Acta Orthop Scand 66: 395-397.

2. Tuček M, Chochola A, Klika D, et al. (2017) Epidemiology of scapular fractures. Acta Orthop Belg 83: 8-15.

3. Ada JR, Miller ME (1991) Scapular fractures. Analysis of 113 cases. Clin Orthop Relat Res 269: 174-180.
4. Cole PA, Gauger EM, Herrera DA, et al. (2012) Radiographic follow-up of 84 operatively treated scapula neck and body fractures. Injury 43: 327-333.

5. Porte AN, Wirtzfeld DA, Mann C (2009) Intrathoracic scapular impaction: an unusual complication of scapular fractures. Can J Surg 52: 62-63.

6. Schwartzbach CC, Seoudi $H$, Ross AE, et al. (2006) Fracture of the scapula with intrathoracic penetration in a skeletally mature patient. A case report. J Bone Joint Surg Am 88: 2735-2738.

7. van Schie-van der Weert EM, van Laanen JH, Kraan GA, et al. (2012) Intrathoracic displacement of a scapular fracture: a case report. J Bone Joint Surg Am 94: e16.

8. Demirkiran ND, Kar A (2016) Pure Intrathoracic Scapular Dislocation. Am J Orthop (Belle Mead NJ) 45: E29-E30.

9. Zlowodzki M, Bhandari M, Zelle BA, et al. (2006) Treatment of scapula fractures: Systematic review of 520 fractures in 22 case series. J Orthop Trauma 20: 230-233.

10. Brandsema B, Neuhaus V, Gradl G, et al. (2016) Extra-articular scapular fractures: Comparison of theoretical and actual treatment. Shoulder Elbow 8: 3-8. 